\title{
ORIGINAL
}

\section{Beta cell dysfunction and its clinical significance in gestational diabetes}

\author{
Yoshifumi Saisho')*, Kei Miyakoshi'²*, Mamoru Tanaka²), Akira Shimada ${ }^{3)}$, Satoru Ikenoue ${ }^{2)}$, \\ Ikuko Kadohira ${ }^{2)}$, Yasunori Yoshimura ${ }^{2)}$ and Hiroshi Itoh ${ }^{1)}$ \\ ${ }^{1)}$ Department of Internal Medicine, Keio University School of Medicine, Tokyo 160-8582, Japan \\ ${ }^{2)}$ Department of Obstetrics and Gynecology, Keio University School of Medicine, Tokyo 160-8582, Japan \\ ${ }^{3)}$ Department of Internal Medicine, Saiseikai Central Hospital, Tokyo 108-0073, Japan
}

\begin{abstract}
The aim of this study is to explore beta cell dysfunction and its clinical significance in gestational diabetes mellitus (GDM). We assessed insulin sensitivity and insulin secretion in a total of 277 Japanese women between 24 and 27 weeks of pregnancy who underwent a $2 \mathrm{~h}, 75 \mathrm{~g}$ oral glucose tolerance test (OGTT) because of an abnormal result on a $1 \mathrm{~h} 50 \mathrm{~g}$ oral glucose challenge conducted as part of a standard screening for GDM. Insulin sensitivity was evaluated by an insulin sensitivity index derived from OGTT $\left(\right.$ IS $_{\text {OGTT }}$ ), whereas insulin secretion was calculated as a ratio of the total area under the insulin curve to the total area under the glucose curve $\left(\mathrm{AUC}_{\mathrm{ins} / \mathrm{glu}}\right)$. Beta cell function in relation to insulin sensitivity (i.e. disposition index) was derived from the product of insulin sensitivity and insulin secretion (i.e. AUC $_{\text {ins/glu }}$ $\left.\times \mathrm{IS}_{\mathrm{OGTT}}\right)$. In women diagnosed with GDM $(\mathrm{n}=57)$, the disposition index was significantly lower than that in those without GDM, irrespective of obesity. The disposition index in women with GDM was significantly correlated with levels of fasting and mean preprandial capillary glucose and HbA1c before initiating insulin therapy $(r=-0.45,-0.38,-0.49$, respectively). Furthermore, there was a significant correlation between the disposition index and total insulin dosage to achieve glycemic goal $(r=-0.41)$. In conclusion, we demonstrated beta cell dysfunction in Japanese women with GDM irrespective of obesity. The level of beta cell dysfunction in GDM was associated with the severity of glucose intolerance and total insulin dosage required. These findings underpin clinical significance of beta cell dysfunction in GDM.
\end{abstract}

Key words: Insulin secretion, Insulin sensitivity, Disposition index, Glucose metabolism, Asian

PANCREATIC BETA CELLS compensate for changes in whole body insulin sensitivity through an increase in insulin secretion. The relationship between insulin sensitivity and insulin secretion is characterized by a rectangular hyperbolic function, such that the product of the two variables, termed as the disposition index, should yield a constant for a given degree of glucose tolerance [1-2]. Although beta cell dysfunction and insulin resistance may occur independently, the disposition index typically decreases through the progression from normal glucose toler-

Received Aug. 4, 2010; Accepted Aug. 20, 2010 as K10E-231

Released online in J-STAGE as advance publication Sep.14, 2010

Correspondence to: Yoshifumi Saisho, M.D., Ph.D., Department of Internal Medicine, Keio University School of Medicine, 35 Shinanomachi, Shinjukuku, Tokyo 160-8582, Japan.

E-mail: saish@sc.itc.keio.ac.jp

*Contributed equally to this study. ance to diabetes. Reduced disposition index reflects deterioration in beta cell compensation for insulin sensitivity [1-2].

During pregnancy, beta cells normally increase their insulin secretion to compensate for a decrease in insulin sensitivity [3-4]. Therefore, a potential etiology for gestational diabetes mellitus (GDM) is a limitation in beta cell reserves [1-3, 5]. Available evidence with regard to beta cell dysfunction in GDM is based on studies conducted in Caucasian and Hispanic women. Asian subjects are classified as a genetically highly susceptible to glucose intolerance (i.e. GDM, type 2 diabetes, and impaired glucose tolerance) [6-9]. Since Asians are less obese than Caucasians, its metabolic features might be different from those found in Caucasians [10-11]. To the best of our knowledge, however, little evidence is available with regard to beta cell function in Asian pregnant women. In addi- 
tion, the clinical impact of beta cell dysfunction on the severity of glucose intolerance in women with GDM remains unknown. Therefore, we performed a retrospective cohort study to address the following questions: (i) Does the hyperbolic insulin sensitivity-secretion relationship exist in Japanese pregnant women? (ii) Is beta cell dysfunction present in Japanese women with GDM irrespective of obesity? (iii) Does beta cell dysfunction reflect the severity of glucose intolerance in women with GDM?

\section{Materials and Methods}

\section{Subjects}

We conducted a retrospective cohort study of 277 consecutive Japanese pregnant women who underwent a diagnostic $2 \mathrm{~h}, 75 \mathrm{~g}$ oral glucose tolerance test (OGTT) because of an abnormal result on a standard $1 \mathrm{~h}, 50 \mathrm{~g}$ oral glucose challenge test (GCT) from 2004 to 2009. All women were cared for at the perinatal unit of Keio University Hospital. Gestational age was confirmed in the first trimester by crown-rump length measurements. Excluded from this study were women with multiple pregnancies and women whose neonates exhibited congenital anomalies. Women with a medical history indicating either impaired glucose tolerance or diabetes mellitus, or the use of medications known to affect glucose metabolism were also excluded. The research was performed in accordance with the Declaration of Helsinki and informed consent was obtained from patients where appropriate. The study was approved by the institutional review board at Keio University School of Medicine.

\section{GDM screening and glucose tolerance status}

Preceding the diagnostic OGTT, each subject underwent a standard 50 g GCT between 24 and 27 weeks of gestation as part of a universal screening procedure for GDM. A venous blood sample was obtained $1 \mathrm{~h}$ after ingestion of a $50 \mathrm{~g}$ oral glucose load, administered without regard for the fasting or fed state. If the GCT result exceeded $140 \mathrm{mg} / \mathrm{dL}$, a $2 \mathrm{~h}$, 75 g OGTT was performed as a diagnostic test [12]. The OGTT was performed after a $12 \mathrm{~h}$ overnight fast. Venous blood samples for measurement of plasma glucose levels and insulin concentrations were drawn in the fasting state and at $30 \mathrm{~min}, 1 \mathrm{~h}$ and $2 \mathrm{hrs}$ after ingestion of the glucose drink.

Based on the criteria using the OGTT proposed by the Japan Society of Obstetrics and Gynecology (JSOG), GDM was diagnosed if two or more values reached or exceeded the following thresholds: fasting, $100 \mathrm{mg} / \mathrm{dL} ; 1$ h, $180 \mathrm{mg} / \mathrm{dL} ; 2$ hrs, $150 \mathrm{mg} / \mathrm{dL}$ [13]. Plasma glucose and insulin levels were measured as previously described by a glucose oxidase method and enzyme immunoassay, respectively [14]. The OGTT result was considered normal if plasma glucose levels in the fasting state and at $1 \mathrm{~h}$ and 2 hrs during the OGTT were less than the thresholds of the JSOG criteria. The normal glucose tolerance (NGT) group comprised women with normal OGTT results.

\section{Daily glucose profile and management of GDM}

All women found to have GDM were hospitalized to undergo dietary management (daily calorie intake: $30 \mathrm{kcal} / \mathrm{kg}+350 \mathrm{kcal}$; if obese, $30 \mathrm{kcal} / \mathrm{kg}$ ). On Day 3 after admission, daily capillary glucose profiles were obtained seven times a day under dietary management: fasting (7:30), $2 \mathrm{~h}$ postbreakfast (10:00), before lunch (11:30), 2 h postlunch (14:00), before dinner (16:30), 2 h postdinner (19:00) and bedtime (21:00). Capillary glucose levels were measured by bedside glucose monitor (Antsense II, HORIBA, Kyoto, Japan). HbA1c levels were measured by HPLC and expressed as an international standard value. Insulin therapy was initiated when dietary treatment did not consistently maintain fasting and premeal capillary glucose $\leq 100 \mathrm{mg} / \mathrm{dL}$ and $2 \mathrm{~h}$ postprandial capillary glucose $\leq 120 \mathrm{mg} / \mathrm{dL}$, respectively. Regular and NPH insulin were used to achieve the glycemic goal and insulin dose was adjusted according to insulin algorithm based on bedside glucose monitor or self-monitoring capillary glucose values. The patients were discharged after titrating the dosage, with the total insulin dosage at discharge being used for analyzing its correlation with beta cell function.

\section{Insulin sensitivity and beta cell function}

Insulin sensitivity and insulin secretion were evaluated using measurements from the diagnostic OGTT. The insulin sensitivity was estimated by the wholebody insulin sensitivity index derived from the OGTT (IS OGTT $_{\text {OG }}$ ) as proposed by Matsuda and Defronzo [15$16]$. The $\mathrm{IS}_{\mathrm{OGTT}}$ is calculated by the following formula: 10,000 / square root $\left\{\mathrm{Glu}_{0} \times \mathrm{Ins}_{0} \times\left(\mathrm{Glu}_{0}+\mathrm{Glu}_{60} \times 2+\right.\right.$ $\left.\left.\mathrm{Glu}_{120}\right) / 2 \times\left(\mathrm{Ins}_{0}+\mathrm{Ins}_{60} \times 2+\mathrm{Ins}_{120}\right) / 2\right\}$, where Glu and Ins $_{\mathrm{y}}$ represent plasma glucose $(\mathrm{mg} / \mathrm{dL})$ and insulin values $(\mu \mathrm{U} / \mathrm{mL})$, respectively, at time y min during 
Table 1 Maternal demographic characteristics of the subjects stratified by glucose tolerance status and the presence of obesity.

\begin{tabular}{|c|c|c|c|c|}
\hline & Non-obese NGT & Non-obese GDM & Obese NGT & Obese GDM \\
\hline $\mathrm{N}$ & 194 & 40 & 26 & 17 \\
\hline Age (years) & $35 \pm 5$ & $36 \pm 4$ & $36 \pm 4$ & $36 \pm 5$ \\
\hline Parous (\%) & 29.4 & 23.1 & 42.3 & 25.0 \\
\hline Prior GDM (\%) & 0 & 7.5 & 8.3 & 0 \\
\hline Family history of diabetes (\%) & 7.7 & 15.0 & 7.7 & 11.8 \\
\hline Gestational weeks at the OGTT & $28 \pm 2$ & $28 \pm 2$ & $28 \pm 4$ & $28 \pm 4$ \\
\hline Pregravid BMI & $20.4 \pm 2.0$ & $19.9 \pm 2.2$ & $27.6 \pm 2.4^{*}$ & $27.6 \pm 1.9 *$ \\
\hline Pregravid body weight (kg) & $51.7 \pm 5.6$ & $49.4 \pm 7.2$ & $68.3 \pm 9.1^{*}$ & $68.6 \pm 5.5^{*}$ \\
\hline Body weight at the OGTT & $57.1 \pm 5.8$ & $54.6 \pm 6.4$ & $70.7 \pm 7.6^{*}$ & $73.5 \pm 6.6^{*}$ \\
\hline
\end{tabular}

NGT: normal glucose tolerance; GDM: gestational diabetes mellitus. *: $p<0.0001$, vs. non-obese women with NGT or GDM.

Table $275 \mathrm{~g}$ OGTT profiles of the subjects stratified by glucose tolerance status and the presence of obesity.

\begin{tabular}{|c|c|c|c|c|}
\hline & Non-obese NGT & Non-obese GDM & Obese NGT & Obese GDM \\
\hline $\mathrm{N}$ & 194 & 40 & 26 & 17 \\
\hline \multicolumn{5}{|c|}{ OGTT-glucose (mg/dL) } \\
\hline $0 \mathrm{~min}$ & $79 \pm 6$ & $83 \pm 8^{*}$ & $82 \pm 7$ & $93 \pm 11 \S \Phi \neq$ \\
\hline $30 \mathrm{~min}$ & $132 \pm 16$ & $159 \pm 20 \S$ & $132 \pm 18 \rrbracket$ & $157 \pm 17 \S \ddagger$ \\
\hline $60 \mathrm{~min}$ & $143 \pm 22$ & $193 \pm 28 \S$ & $146 \pm 179$ & $190 \pm 26 \S \ddagger$ \\
\hline $120 \mathrm{~min}$ & $120 \pm 19$ & $167 \pm 24 \S$ & $124 \pm 14 \uparrow$ & $168 \pm 26 \S \ddagger$ \\
\hline \multicolumn{5}{|c|}{ OGTT-insulin (mU/L) } \\
\hline $0 \mathrm{~min}$ & $6.0 \pm 2.9$ & $7.0 \pm 4.1$ & $9.4 \pm 3.7 \S £$ & $14.6 \pm 5.8 \S$ 粒 \\
\hline $30 \mathrm{~min}$ & $51.8 \pm 28.1$ & $56.4 \pm 28.2$ & $65.6 \pm 32.8$ & $68.5 \pm 26.9$ \\
\hline $60 \mathrm{~min}$ & $63.0 \pm 32.9$ & $73.7 \pm 39.2$ & $80.6 \pm 33.7$ & $108.1 \pm 45.5 \S \#$ \\
\hline $120 \mathrm{~min}$ & $53.5 \pm 31.6$ & $83.9 \pm 49.0$ & $71.2 \pm 46.2$ & $121.2 \pm 48.7 \S \# \dagger$ \\
\hline
\end{tabular}

NGT: normal glucose tolerance; GDM: gestational diabetes mellitus. ${ }^{*}: p<0.001, \S$ : $p<0.0001$ vs. non-obese women with NGT. $£$ : $p<0.05$, \#: $p<0.01$, ๆ: $p<0.0001$ vs. non-obese women with GDM. †: $p<0.001$, $\neq: p<0.0001$ vs. obese women with NGT.

the OGTT. Insulin secretion was assessed by the ratio of the total area under the insulin curve to the total area under the glucose curve (AUC ins/glu $)$ during the OGTT. Beta cell function was assessed by the product of insulin sensitivity and insulin secretion (i.e. the disposition index), as follows: the AUC ins/glu multiplied by IS $_{\text {OGTT }}$.

To investigate the metabolic characteristics (i.e. insulin sensitivity, insulin secretion, and beta cell function) in subjects stratified by glucose tolerance status and anthropometric parameters, subjects were divided into the four subgroups: (i) non-obese women with NGT, (ii) non-obese women with GDM, (iii) obese women with NGT, (iv) obese women with GDM. Since Japanese women tend to be leaner than their
Caucasian counterparts, obesity was defined as body mass index (BMI) $\geq 25$ based on weight and height before pregnancy, as proposed by Japan Society for the Study of Obesity [17].

\section{Statistical analysis}

Data are presented as mean \pm SD in text and tables, and illustrated as mean \pm SEM in figures. In Tables 1 and 2, and Fig. 2, continuous variables were tested for normality of distribution and were compared among four subgroups using the one-way analysis of variance or the Kruskal-Wallis test, followed by Scheffe's post hoc analysis. In Table 1 , categorical variables are presented as proportions and were assessed with the $\chi^{2}$ test or Fisher's exact test. Simple or multiple regres- 
sion analysis was used to test the correlation between the disposition index and the glycemic profiles or total insulin dosage (Fig. 3 and 4). Statistical analysis was performed using StatView (SAS Institute, Cary, NC, USA). Hyperbolic curves were drawn by GraphPad Prism software (GraphPad Software, La Jolla, CA, USA) (Fig. 1). $P<0.05$ was considered as statistically significant.

\section{Results}

\section{Maternal demographic characteristics and $75 \mathrm{~g}$ OGTT profiles}

Demographic characteristics of the subjects stratified by glucose tolerance status and the presence of obesity are shown in Table 1. There were no significant differences between groups with respect to maternal age, gestational weeks at the diagnostic OGTT, parity, and the prevalence of a family history of diabetes and a history of previous GDM. Pregravid BMI in non-obese women with NGT was similar to those with GDM $(20.4 \pm 2.0$ and $19.9 \pm 2.2$, respectively). Likewise, there was no significant difference in pregravid BMI between obese women with NGT and GDM $(27.6 \pm 2.4$ and $27.6 \pm 1.9$, respectively). Since changes in body weight during pregnancy were similar among the groups, neither non-obese nor obese women showed significant differences in body weight at the OGTT between those with NGT and GDM (Table 1).

The 75 g OGTT profiles in each study group are summarized in Table 2. Women with GDM showed significantly higher plasma glucose concentrations at all time points during the OGTT compared to those with NGT in either non-obese or obese subjects ( $p$ $<0.001$ ). Obese women with GDM showed significantly higher fasting glucose concentration than nonobese women with GDM (93 \pm 11 vs. $83 \pm 8 \mathrm{mg} / \mathrm{dL}, p$ $<0.0001$ ), while plasma glucose levels at 30,60 , and 120 min were comparable between the two groups.

There were no significant differences between nonobese women with GDM and NGT regarding plasma insulin levels throughout the OGTT. In contrast, obese women with GDM showed significantly higher plasma insulin concentration at 0 and $120 \mathrm{~min}$, compared with obese women with NGT. Similarly, obese NGT women showed higher fasting plasma insulin concentration than non-obese NGT women (9.4 \pm 3.7 vs. $6.0 \pm 2.9 \mu \mathrm{U} / \mathrm{mL}, p<0.0001)$.
Relationship between insulin sensitivity and insulin secretion in women with NGT or GDM

The relationships between IS $_{\text {OGTT }}$ and AUC ins/glu were non-linear and best described by a hyperbolic function: NGT ( $r=0.82, p<0.0001)$ and GDM ( $r=$ $0.82, p<0.0001$ ) (Fig. 1). In addition, the hyperbolic curve of the women with GDM lay down and to the left from those with NGT.

\section{Insulin sensitivity and beta cell function in women with NGT or GDM}

The $\mathrm{IS}_{\text {OGTT }}$ was highest in non-obese NGT, followed in turn by non-obese GDM, obese NGT, and obese GDM women $(p<0.0001)$ (Fig. 2A). Non-obese women with GDM exhibited lower IS $_{\text {OGTT }}$ values than non-obese women with NGT ( $5.3 \pm 2.2$ vs. $7.7 \pm 4.0$, $p$ $<0.01)$. Although the difference did not reach statisti-

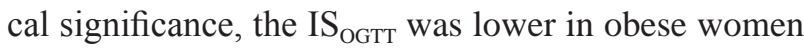
with GDM than in obese women with NGT (2.6 \pm 0.8 vs. $4.8 \pm 1.7, p=0.3$ ).

With regard to insulin secretion, there were no significant differences in the AUC ins/glu between nonobese women with GDM and NGT $(0.40 \pm 0.18 v s$. $0.38 \pm 0.19, p=0.6$ ) (Fig. 2B). Likewise, the $\mathrm{AUC}_{\text {ins/ }}$ glu was similar in obese women with GDM and NGT ( $0.55 \pm 0.20$ vs. $0.51 \pm 0.20, p=0.9)$. The AUC ins/ glu in obese women with GDM and NGT were significantly higher than that in non-obese women, respectively (both $p<0.05$ ). The disposition index in nonobese women with GDM was significantly lower than in non-obese women with NGT $(1.7 \pm 0.4$ vs. $2.6 \pm 1.0$, $p<0.0001$ ) (Fig. 2C). Likewise, obese women with GDM showed lower disposition index compared to those with NGT $(1.3 \pm 0.4 v s .2 .2 \pm 0.5, p<0.01)$. The disposition indices were comparable between nonobese and obese women with NGT $(p=0.07)$.

\section{Daily glycemic profiles, insulin therapy, and beta cell dysfunction in women with GDM}

When the relationship between the disposition index and glycemic profiles was examined, a significant inverse correlation was noted between the disposition index and levels of HbA1c ( $r=-0.49, p=0.0002)$, fasting $(r=-0.45, p=0.0006)$, mean daily and preprandial capillary glucose levels $(r=-0.38$ and $-0.31 ; p=0.005$ and 0.02, respectively) (Fig. 3, A-D). However, the disposition index was not significantly correlated with the mean postprandial capillary glucose levels ( $r$ $=-0.12, p=0.4$ ) (Fig. 3E). Moreover, the disposition 



Fig. 1 Insulin sensitivity-secretion relationship in women with NGT or GDM. (A) Scatterplots of insulin sensitivity determined by $\mathrm{IS}_{\text {OGTT }} v s$ insulin secretion determined by AUC ins/glu in women with NGT (white circle) and GDM (closed triangle). (B) The hyperbolic correlation between $\mathrm{IS}_{\text {OGTT }}$ and AUC ins/glu $_{\text {in }}$ the NGT $(r=0.82, p<0.0001)$ and GDM groups $(r=0.82, p<0.0001)$. The hyperbolic curve in women with GDM was shifted down and to the left compared to that in those with NGT. NGT: normal glucose tolerance, GDM: gestational diabetes mellitus.
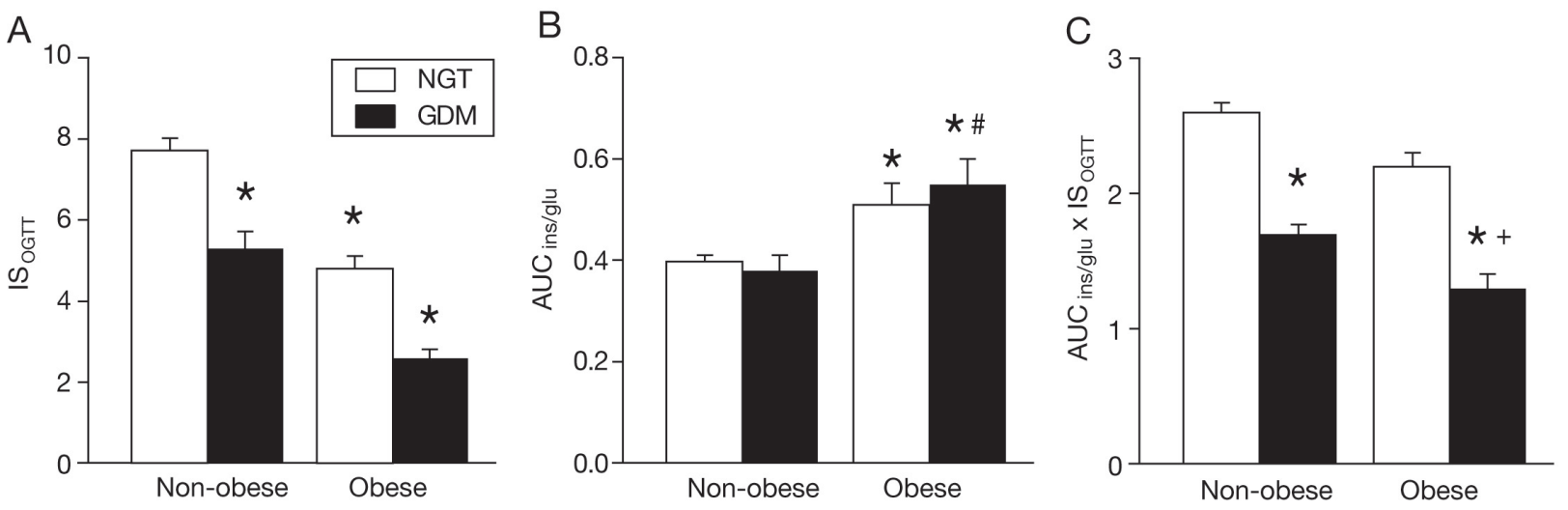

Fig. 2 Insulin sensitivity and beta cell function in women with NGT or GDM. Insulin sensitivity was determined by IS ${ }_{\text {OGTT }}$ (A), insulin secretion determined by AUC ${ }_{\text {ins/glu }}(B)$, and beta cell function quantified as AUC $_{\text {ins/glu }}$ x IS $_{\text {oGTT }}$ (i.e. disposition index) (C) in women stratified by glucose tolerance status and the presence of obesity. Insulin secretion did not increase to compensate for decreased insulin sensitivity in women with GDM compared to NGT in both non-obese and obese individuals. Disposition index in women with GDM was significantly lower than that in those with NGT in both non-obese and obese individuals. *: $p$ $<0.05$ vs. non-obese NGT, \#: $p<0.05$ vs. non-obese GDM, +: $p<0.05$ vs. obese NGT. NGT: open bar, GDM: closed bar.

index was not significantly correlated with coefficient of variation (CV) of daily capillary glucose levels $(r=$ $0.15, p=0.3$ ) (Fig. 3F). Of 57 women with GDM, 43 women (non-obese, $n=27$; obese, $n=16$ ) needed insulin therapy to achieve the glycemic goal (total insulin dosage: $25 \pm 20$ units daily; regular insulin: $19 \pm 13$ units daily, NPH insulin: $5 \pm 10$ units daily). The disposition index was inversely correlated with the total dosage of insulin needed to attain the defined glycemic target ( $r$ $=-0.42, p=0.006$, Fig. 4A). This correlation persisted after adjustment for body weight $(r=-0.40, p=0.008$, Fig. 4B). Furthermore, multiple regression analysis revealed that the disposition index was independently correlated with insulin dose after adjustment for BMI $(r=-0.36, p=0.02)$.

\section{Discussion}

The current study demonstrates a hyperbolic relationship exists between insulin sensitivity and insulin 

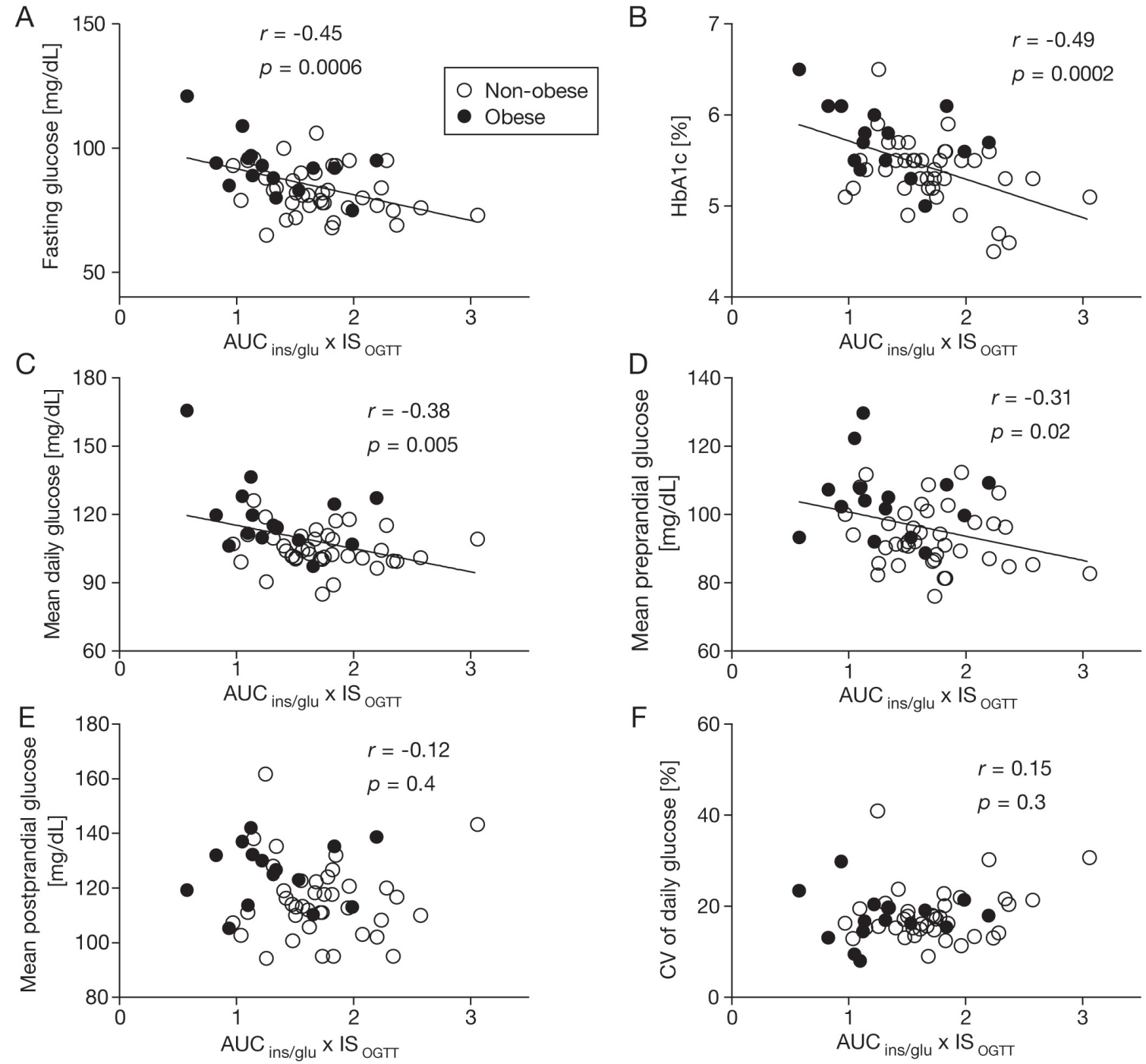

Fig. 3 Relationship between disposition index (AUC ins/glu $\times$ IS $_{\text {OGTT }}$ ) and blood glucose levels in women with GDM. Note that the disposition index was significantly correlated with levels of fasting and the mean daily and preprandial capillary glucose, and HbA1c. Non-obese GDM: open circle, Obese GDM: closed circle.
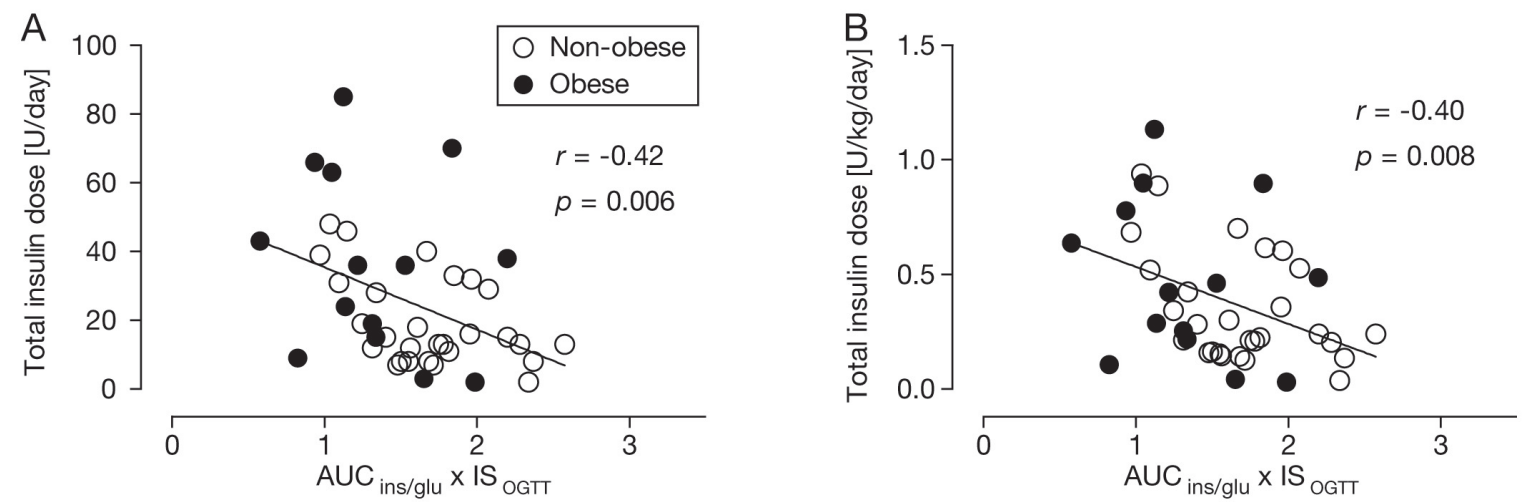

Fig. 4 Relationship between disposition index $\left(\mathrm{AUC}_{\mathrm{ins} / \mathrm{glu}} \times \mathrm{IS}_{\mathrm{OGTT}}\right)$ and total insulin dosage needed in women with GDM (A: U/day, $\mathrm{B}$ : $\mathrm{U} / \mathrm{kg} / \mathrm{day}, \mathrm{n}=43$ ). The disposition index was significantly correlated with total insulin dosage to achieve glycemic control. Non-obese GDM: open circle, Obese GDM: closed circle. 
secretion in Japanese pregnant women. The product of insulin sensitivity and insulin secretion (i.e. disposition index), a measure of beta cell function in relation to insulin sensitivity, was low in women with GDM compared with those with NGT both in obese and non-obese subgroups. Moreover, beta cell dysfunction in women with GDM was associated with fasting and the mean daily capillary glucose levels and insulin dosage needed to achieve good glycemic control. To the best of our knowledge, this is the first report to demonstrate beta cell dysfunction and its clinical significance in Asian women with GDM.

The AUC ins/glu and IS $_{\text {OGTT }}$ obtained from OGTT have been widely used and well validated $[16,18]$. Similar to previous data in Caucasian subjects, we found that the hyperbolic curve in women with GDM lay down and to the left compared with the women with NGT. Consistent with this observation, the disposition index (i.e. AUC ins/glu $\times \mathrm{IS}_{\mathrm{OGTT}}$ ) in women with GDM was significantly lower than that in those with NGT. Therefore, our results indicate beta cell dysfunction in Japanese women with GDM irrespective of presence or absence of obesity.

Despite less obesity, the incidence of type 2 diabetes in Japanese subjects is similar to that in the United Sates, suggesting Japanese have less beta cell functional capacity compared to Caucasians [10]. Additionally, Japanese seem to be more susceptible to obesity-induced glucose intolerance than Caucasians [17]. To date, most studies on beta cell function in GDM were performed in a cohort of obese Caucasian and Hispanic subjects. Therefore, Japanese subjects are useful to investigate the presence of beta cell dysfunction in nonobese as well as obese subjects with GDM.

Since the women with NGT in this study had an abnormal GCT value, the $\sim 40 \%$ decrease in the disposition index in women with GDM may be underestimat- ed. Consistent with previous studies, we also found decreased insulin sensitivity in women with GDM compared to BMI-matched women with NGT [3-5]. However, it remains unknown whether it is due to a primary defect or is secondary to hyperglycemia.

In women with GDM, the lower the disposition index, the higher the fasting and mean preprandial plasma glucose and HbA1c. Likewise, the disposition index was inversely correlated with total insulin dosage to achieve good glycemic control irrespective of obesity. These results suggest that the severity of the glucose intolerance in women with GDM reflects the degree of their beta cell dysfunction. Of interest, the disposition index was not associated with postprandial and CV and the standard deviation of daily capillary glucose levels in our study (data on the standard deviation of daily capillary glucose levels not shown). These findings might be because these plasma glucose levels were measured after meal ingestion, but not glucose ingestion.

In conclusion, women with GDM have beta cell dysfunction irrespective of presence or absence of obesity. In women with GDM, beta cell dysfunction was associated with increased fasting and mean daily capillary glucose levels and total insulin dosage needed to achieve good glycemic control. These findings underpin the clinical significance of beta cell dysfunction in GDM.

\section{Acknowledgement}

The authors acknowledge all the women who participated in this study, and medical staff in the perinatal unit of Keio University Hospital for excellent patient care. The authors have no conflict of interest to disclose. We thank Dr. Jon Hennebold for his helpful suggestions in preparing the manuscript.

\section{References}

1. Bergman RN, Ader M, Huecking K, Van Citters G (2002) Accurate assessment of beta-cell function: the hyperbolic correction. Diabetes 51 Suppl 1: S212-220.

2. Buchanan TA (2001) Pancreatic B-cell defects in gestational diabetes: implications for the pathogenesis and prevention of type 2 diabetes. J Clin Endocrinol Metab 86: 989-993.

3. Homko C, Sivan E, Chen X, Reece EA, Boden G (2001) Insulin secretion during and after pregnancy in patients with gestational diabetes mellitus. J Clin Endocrinol Metab 86: 568-573.

4. Catalano PM, Tyzbir ED, Wolfe RR, Calles J, Roman NM, Amini SB, Sims EA (1993) Carbohydrate metabolism during pregnancy in control subjects and women with gestational diabetes. Am J Physiol 264: E60-67.

5. Xiang AH, Peters RK, Trigo E, Kjos SL, Lee WP, Buchanan TA (1999) Multiple metabolic defects during late pregnancy in women at high risk for type 2 diabe- 
tes. Diabetes 48: 848-854.

6. Berkowitz GS, Lapinski RH, Wein R, Lee D (1992) Race/ethnicity and other risk factors for gestational diabetes. Am J Epidemiol 135: 965-973.

7. Dabelea D, Snell-Bergeon JK, Hartsfield CL, Bischoff KJ, Hamman RF, McDuffie RS (2005) Increasing prevalence of gestational diabetes mellitus (GDM) over time and by birth cohort: Kaiser Permanente of Colorado GDM Screening Program. Diabetes Care 28: 579-584.

8. Lawrence JM, Contreras R, Chen W, Sacks DA (2008) Trends in the prevalence of preexisting diabetes and gestational diabetes mellitus among a racially/ethnically diverse population of pregnant women, 1999-2005. Diabetes Care 31: 899-904.

9. Araneta MR, Wingard DL, Barrett-Connor E (2002) Type 2 diabetes and metabolic syndrome in FilipinaAmerican women : a high-risk nonobese population. Diabetes Care 25: 494-499.

10. Sone H, Ito H, Ohashi Y, Akanuma Y, Yamada N (2003) Obesity and type 2 diabetes in Japanese patients. Lancet 361: 85.

11. Yoon KH, Lee JH, Kim JW, Cho JH, Choi YH, Ko SH, Zimmet P, Son HY (2006) Epidemic obesity and type 2 diabetes in Asia. Lancet 368: 1681-1688.

12. Miyakoshi K, Tanaka M, Ueno K, Uehara K, Ishimoto H, Yoshimura Y (2003) Cutoff value of 1 h, 50 g glu- cose challenge test for screening of gestational diabetes mellitus in a Japanese population. Diabetes Res Clin Pract 60: 63-67.

13. Kuzuya T, Nakagawa S, Satoh J, Kanazawa Y, Iwamoto Y, Kobayashi M, Nanjo K, Sasaki A, Seino Y, Ito C, Shima K, Nonaka K, Kadowaki T (2002) Report of the Committee on the classification and diagnostic criteria of diabetes mellitus. Diabetes Res Clin Pract 55: 65-85.

14. Saisho Y, Maruyama T, Hirose H, Saruta T (2007) Relationship between proinsulin-to-insulin ratio and advanced glycation endproducts in Japanese type 2 diabetic subjects. Diabetes Res Clin Pract 78: 182-188.

15. Matsuda M, DeFronzo RA (1999) Insulin sensitivity indices obtained from oral glucose tolerance testing: comparison with the euglycemic insulin clamp. Diabetes Care 22: 1462-1470.

16. DeFronzo RA, Matsuda M (2010) Reduced time points to calculate the composite index. Diabetes Care 33: e93.

17. Examination Committee of Criteria for 'Obesity Disease' in Japan (2002) New criteria for 'obesity disease' in Japan. Circ J 66: 987-992.

18. Retnakaran R, Shen S, Hanley AJ, Vuksan V, Hamilton JK, Zinman B (2008) Hyperbolic relationship between insulin secretion and sensitivity on oral glucose tolerance test. Obesity (Silver Spring) 16: 1901-1907. 